\title{
Giant Oral Ulcers Following Suicide Attempt by Paraquat Herbicide
}

\author{
Reza Bidaki ${ }^{1,2}$, Seyed Nader Mostafavi ${ }^{3}$, Sogol Al-Saeed ${ }^{3}$, Mojtaba Babaei Zarch ${ }^{3}$, Motahhareh \\ Karimoddini ${ }^{3}$, Farzaneh Dehghani ${ }^{*}$
}

'Department of Psychiatry, Research Center of Addiction and Behavioral Sciences, Shahid Sadoughi University of Medical Sciences, Yazd, Iran

${ }^{2}$ Diabetes Research Center, Shahid Sadoughi University of Medical Sciences, Yazd, Iran

${ }^{3}$ Shahid Sadoughi University of Medical Sciences, Yazd, Iran

*Corresponding Author: Farzaneh Dehghani, M.D., Psychiatry Resident, Department of Psychiatry, Research Center of Addiction and Behavioral Sciences, Shahid Sadoughi University of Medical Sciences, Yazd, Iran. Tel: +98-3536232003, Fax: +98-3532633555, Email: dehghani.med@gmail.com

Received April 26, 2020; Accepted August 16, 2020; Online Published August 21, 2020

\begin{abstract}
Introduction: Parquet poisoning has been elaborated to be very common among developing countries, particularly in regions with agriculture economy. One of the most common presentations of paraquat poisoning is oropharyngeal burns. Herein, we report a patient from a dry and warm district in the geographic center of Iran where agriculture and, as a result, paraquat poisoning are not commonly reported.

Case Presentation: A 25-year-old man presented to hospital subsequent to a suicide attempt by ingesting oral paraquat poison. He gradually developed odynophagia and trismus-like appearance that, after a psychiatric consultation, was misdiagnosed as a drug side effect in the form of dystonia. Upon further physical examination, oral ulcers on his tongue were revealed to be responsible for the symptom.

Conclusion: Paraquat poisoning is very rare in regions with lower rates of agriculture activities. In any patient with poisoning, however, it is necessary to consider intraoral examination to rule out any other suspected diagnosis.

Keywords: Oral Ulcer, Suicide, Paraquat Poisoning
\end{abstract}

\section{Introduction}

Paraquat poison (N,N'-dimethyl-4,4'-bipyridinium dichloride) is one of the most widely used herbicides in the world. Herbicide poisoning has been elaborated to be very common in developing countries, particularly in regions with agriculture economies. ${ }^{1-3}$ Thirty $\mathrm{mg} / \mathrm{kg}$ paraquat intake is considered a lethal dose. Depending on the amount of paraquat ingested, the signs and symptoms vary from mild to moderate to fulminant. Multi-organ failure is likely to occur with ingestion of $\geq 50 \mathrm{mg} / \mathrm{kg}$ of the poison, which may cause death within 3 days. ${ }^{4-6}$ Other common presentations of paraquat poisoning could be manifested by minor gastrointestinal problems like transient vomiting, diarrhea, and oropharyngeal burns. ${ }^{2,6-8}$

Herein, we report a patient from a dry and warm district in the geographic center of Iran where agriculture and, as a result, paraquat poisoning are not commonly reported.

\section{Case Presentation}

A 25-year-old married man presented to emergency medical services less than 2 hours after orally ingesting
6 grams of paraquat poison. He attempted an impulsive suicide after marital discord by deliberately consuming paraquat.

Immediately after ingestion of the herbicide, the patient experienced nausea, vomiting, and abdominal pain. His vital signs were normal at admission; thereafter, he developed drowsiness without loss of consciousness. In less than 3 days, oral ulcers appeared on the patient's tongue; he experienced odynophagia and was unable to open his mouth because of sore throat. The patient could barely swallow or chew which could represent trismus. Intraoral examination revealed a large ulcer with a white necrotic base covering two thirds of the tongue (Figure 1). Bleeding from the surface was seen between the areas of necrosis.

The patient received fluid therapy, antiemetic, intravenous vitamin E 200 IU/daily, spray lidocaine, and $\mathrm{H} 2$ blockers. Based on lab data, the patient underwent 9 sessions of hemodialysis due to acute renal failure. The lab data revealed abnormalities as shown in Table 1.

For tongue burn, topical application of metronidazole oral gel was prescribed to prevent secondary infection. The

Copyright $\odot 2020$ The Author(s). This is an open-access article distributed under the terms of the Creative Commons Attribution License (http:// creativecommons.org/licenses/by/4.0), which permits unrestricted use, distribution, and reproduction in any medium, provided the original work is properly cited. 


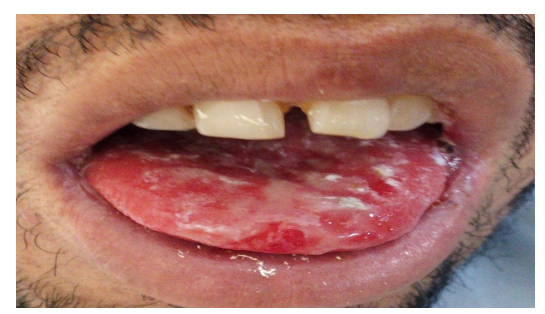

Figure 1. Giant and Extensive Oral Ulcers Following Oral Ingestion of Paraquat.

necessity of serial monitoring for renal, hepatic, and lung injuries and follow-up were justified for the patient after discharge.

During hospitalization, psychiatry consultation for substance use disorder was considered for the patient based on the Diagnostic and Statistical Manual of Mental Disorders (DSM-5), because the patient reported smoking $0.5 \mathrm{mg}$ opium per day with cigarettes. He was a sensitive person and had poor coping mechanisms, and he had previously attempted suicide with benzodiazepine. Based on his recent marital discord, adjustment disorder was also probable for him. Ultimately, couple therapy and pharmacotherapy were started for the patient.

\section{Discussion}

Suicide attempt with herbicide is not only attributed to developing countries. ${ }^{9}$ In a certain period of time, 56\% of all human deaths from pesticides in England was attributed to paraquat. In 2008, paraquat more than any other pesticide was responsible for deaths in the United States. ${ }^{10}$ Nonetheless, paraquat poisoning has not been previously reported in the geographic center of Iran where agriculture activities are not common. Once the patient reported herein was evaluated in his first psychiatry consult, severe muscular spasm in his jaws was the most predominant presentation. Drug-induced dystonia was suspected as the first differential diagnosis, but further exploration revealed giant oral ulcers throughout his buccal area and tongue, which gave him trismus. In the current case, serum paraquat poison levels were not available for confirmation. This common laboratory deficit in addition to less-frequent reports on paraquat poisoning may lead to misdiagnoses and eventual underestimation of the number of poisoning incidences. Therefore, physical examination plays an important role in identifying such cases. As reported in previous paraquat poisoning case reports, ${ }^{11,12}$ paraquat poisoning by oral ingestion may have varying effects on oral mucosa and the tongue, which may differ from pseudo diphtheria to prominent pharyngeal membrane ${ }^{11,13}$; to paraquat tongue to large and extensive oral ulcers (Figure 1). This may, in turn, give the mouth a trismus-like appearance.

Mucosal lesions of the mouth and tongue, however, cannot specifically predict the severity of poisoning. Mucosal lesions in the pharynx, esophagus, and gastric area are also very common and much more ominous. These can eventuate in perforation, mediastinitis, and/

\begin{tabular}{lcc} 
Table 1. Laboratory data & & \\
\hline $\mathbf{W B C}=\mathbf{2 3 0 0}$ & $\mathbf{H b}=\mathbf{1 1}$ & Platelet $\downarrow$ \\
\hline $\mathrm{FBS}=238 \uparrow$ & $\mathrm{K}=3.4$ & $\mathrm{ESR}=86$ \\
$\mathrm{ALT} \uparrow$ & $\mathrm{Na}=132$ & $\mathrm{U} / \mathrm{A}: \mathrm{NL}$ \\
$\mathrm{AST} \uparrow$ & Creatinine $=2.4$ & \\
ALKP $\uparrow \uparrow$ & $\mathrm{BUN}=89$ & \\
\hline
\end{tabular}

or pneumomediastinum. ${ }^{2}$ The mortality rate of paraquat poisoning in suicide attempts is about $70 \%$; that percentage accounts for the acute systemic effects presented by pulmonary edema, convulsion, and cardiac, renal, and hepatic failure. ${ }^{14}$ According to the lab data, hepatic failure, thrombocytopenia, and also bleeding were ruled out, and dialysis was ordered to hinder renal failure. Despite the high probability of death for the current case, the patient survived this situation.

\section{Conclusion}

Paraquat poisoning is very rare in regions with lower rates of agricultural activities. In any patient with poisoning, however, it is necessary to consider intraoral examination to rule out any other suspected diagnoses.

\section{Authors' Contributions}

All authors contributed equally to current case report.

\section{Conflict of Interest Disclosures}

The authors declare that they have no conflicts of interest.

\section{Ethical Approval}

The authors obtained permission from the patient to report this case.

\section{Acknowledgments}

The authors hereby acknowledge the patient for participating in this study and the authors' collaboration in preparing this paper.

\section{References}

1. Hong SY, Hwang KY, Lee EY, et al. Effect of vitamin C on plasma total antioxidant status in patients with paraquat intoxication. Toxicol Lett. 2002;126(1):51-59. doi:10.1016/ s0378-4274(01)00431-3.

2. Gawarammana IB, Buckley NA. Medical management of paraquat ingestion. Br J Clin Pharmacol. 2011;72(5):745-757. doi:10.1111/j.1365-2125.2011.04026.x.

3. Wesseling C, van Wendel de Joode B, Ruepert C, et al. Paraquat in developing countries. Int J Occup Environ Health. 2001;7(4):275-286. doi:10.1179/107735201800339209.

4. Dinis-Oliveira RJ, Duarte JA, Sánchez-Navarro A, Remião F, Bastos ML, Carvalho F. Paraquat poisonings: mechanisms of lung toxicity, clinical features, and treatment. Crit Rev Toxicol. 2008;38(1):13-71. doi:10.1080/10408440701669959.

5. Fernando MBJ, Camilo MOJ, Pérez-Nieto OR, Jesús RC. Paraquat intoxication as a cause of multiple organ failure: report of a case and review of the literature. MOJ Toxicol. 2018;4(4):247-253. doi:10.15406/mojt.2018.04.00108.

6. Isha IT, Alam Z, Shaha BK, Bari MS, Bari MZJ, Chowdhury 
FR. Paraquat induced acute kidney injury and lung fibrosis: a case report from Bangladesh. BMC Res Notes. 2018;11(1):344. doi:10.1186/s13104-018-3425-3.

7. Ackrill P, Hasleton PS, Ralston AJ. Oesophageal perforation due to paraquat. Br Med J. 1978;1(6122):1252-1253. doi:10.1136/ bmj.1.6122.1252.

8. Rahmani AH, Forouzandeh $\mathrm{H}$, Tadayon Khatibi M. Medical management and outcome of paraquat poisoning in Ahvaz, Iran: a hospital-based study. Asia Pac J Med Toxicol. 2015;4(2):74-78. doi:10.22038/apjmt.2015.5086.

9. Raghu K, Mahesh V, Sasidhar P, Reddy PR, Venkataramaniah V, Agrawal A. Paraquat poisoning: a case report and review of literature. J Family Community Med. 2013;20(3):198-200. doi:10.4103/2230-8229.122023.
10. Jeyaratnam J. Acute pesticide poisoning: a major global health problem. World Health Stat Q. 1990;43(3):139-144.

11. Madhan B, Arunprasad G, Krishnan B. Paraquat tongue. BMJ Case Rep. 2014;2014. doi:10.1136/bcr-2014-206581.

12. 13 Patel RK, Sa DK, Behra A, Meher K. A rare case of "paraquat tongue". Indian J Dermatol. 2020;65(3):245-246. doi:10.4103/ ijd.IJD_659_18.

13. Stephens DS, Walker DH, Schaffner W, et al. Pseudodiphtheria: prominent pharyngeal membrane associated with fatal paraquat ingestion. Ann Intern Med. 1981;94(2):202-204. doi:10.7326/0003-4819-94-2-202.

14. Chen H-W, Tseng T-K, Ding L-W. Intravenous paraquat poisoning. J Chin Med Assoc. 2009;72:547-550. doi:10.1016/ S1726-4901(09)70426-5 